\title{
Cierre de túnel aorto-ventricular derecho mediante un dispositivo percutáneo
}

\author{
Closure of the right aorto-ventricular tunnel through a percutaneous device
}

\author{
María Loo-Valverde ${ }^{1,2 *}$, Vivian Tuesta-García ${ }^{1}$, Benigno Reyes-Vega ${ }^{1}$, Lucy E. Correa-López ${ }^{2}$ y \\ Alonso Soto ${ }^{2,3}$
}

${ }^{1}$ Departamento de Pediatría Clínica, Servicio de Pediatría Especializada, Unidad de Cardiología Pediátrica, Hospital Nacional Edgardo Rebagliati Martins; ${ }^{2}$ Instituto de Investigación en Ciencias Biomédicas (INICIB), Universidad Ricardo Palma; ${ }^{3}$ Departamento de Medicina Interna, Hospital Nacional Hipólito Unanue. Lima, Perú

\section{Resumen}

El túnel aorto-ventricular derecho es una anomalía congénita que consiste en un canal que conecta la aorta ascendente a la cavidad del ventrículo derecho. El diagnóstico se corrobora, en la mayoría de los casos, por ecocardiografía. El cierre quirúrgico se considera el tratamiento de elección y solo se han reportado dos casos previos en los que se ha logrado el cierre mediante intervencionismo. Se presenta el caso de una lactante con diagnóstico de túnel aorto-ventricular derecho, tratada por intervención percutánea con dispositivo de cierre percutáneo, que tuvo buena evolución clínica. La paciente, además, presentó un ductus permeable, el cual fue tratado en una segunda oportunidad por intervencionismo. La factibilidad de la técnica de cierre por procedimiento percutáneo y su baja tasa de complicaciones en comparación con el cierre por cirugía abierta pueden hacer de este el procedimiento de elección en la mayoría de los casos para esta infrecuente condición.

Palabras clave: Túnel aorto-ventricular derecho. Ventrículo derecho. Cardiopatía congénita.

\section{Abstract}

The aorto-right ventricular tunnel is a congenital anomaly that consists of a channel that connects the ascending aorta to the right ventricular cavity. The diagnosis is usually made by transthoracic echocardiography. Surgical closure is usually considered the treatment of choice and only two previous cases have been reported in which closure has been achieved by percutaneous intervention. We report a case of an infant with a diagnosis of aorto-right ventricular tunnel treated using a percutaneous device with good clinical evolution. The patient had also a patent ductus which was later treated by interventionism. The feasibility of the technique and its low rate of complications compared to the closure by open surgery can make it the procedure of choice in most cases for this rare condition.

Key words: Aorto-right ventricular tunnel. Right ventricle. Congenital heart disease.

\section{Correspondencia:}

*María Loo-Valverde

E-mail: maryloovalverde@gmail.com
Fecha de recepción: 17-02-2020

Fecha de aceptación: 05-08-2020

DOI: 10.24875/RCCAR.M21000069
Disponible en internet: $18-10-2021$ Rev Colomb Cardiol. 2021;28(4):378-382 www.rccardiologia.com 0120-5633 / @ 2020 Sociedad Colombiana de Cardiología y Cirugía Cardiovascular. Publicado por Permanyer. Este es un artículo open access bajo la licencia CC BY-NC-ND (http://creativecommons.org/licenses/by-nc-nd/4.0/). 


\section{Introducción}

El túnel aorto-ventricular es una malformación congénita rara. Usualmente, la comunicación se establece con el ventrículo izquierdo. El túnel aorto-ventricular derecho es aún más infrecuente, y consiste en una comunicación entre la aorta ascendente, por encima de la unión sinotubular y el ventrículo derecho ${ }^{1}$. En pacientes con cardiopatía congénita tiene una incidencia del $0.001 \%^{2}$ y se presenta con mayor frecuencia en varones.

Desde el punto de vista embriológico, se han propuesto varias teorías sobre el desarrollo de esta malformación, entre ellas la incorporación defectuosa en el extremo distal del bulbus cordis ${ }^{3}$ y conexiones fistulosas de la arteria coronaria en el ventrículo ${ }^{4}$. El origen del desarrollo de un túnel podría estar relacionado con la regresión del músculo que separa con un plano de tejido extracardiaco a los senos de las válvulas aórtica y pulmonar con los cojinetes endocárdicos. En algunas ocasiones, las arterias coronarias no se conectan con los senos aórticos debido al desarrollo anormal de este tejido extracardiaco ${ }^{5}$.

Aproximadamente en el $80 \%$ de los casos, el túnel aorto-ventricular se origina por encima de la coronaria derecha, recorriendo el plano de tejido entre la aorta y el infundíbulo pulmonar, para incorporarse al ventrículo izquierdo. Casi el $90 \%$ de los túneles terminan en el ventrículo izquierdo; es mucho más raro que lo hagan en el derecho. La anomalía produce un shunt hemodinámicamente significativo de izquierda a derecha, que causa sobrecarga de presión y volumen del ventrículo derecho. La mayoría de los pacientes presentan insuficiencia cardiaca congestiva en la infancia, pero esta puede variar desde la muerte fetal hasta un hallazgo en adultos asintomáticos ${ }^{5}$.

Se suele evidenciar un soplo sistodiastólico en foco aórtico, con frémito e irradiación supraesternal, acompañado de pulsos amplios. En los pacientes mayores, los síntomas se asemejan más a los de una insuficiencia valvular aórtica con alteraciones en la presión arterial sistémica. El electrocardiograma y la radiografía de tórax en proyección posteroanterior suelen mostrar hipertrofia ventricular derecha. Puede haber sobrecarga diastólica y cardiomegalia con dilatación de la aorta ascendente. La ecocardiografía transtorácica es la prueba diagnóstica de elección: el eje largo paraesternal y el eje corto demuestran el túnel con dilatación de la aorta ascendente. En el estudio Doppler color se observa flujo diastólico originado en la unión sino-tubular, alejándose hacia el ventrículo izquierdo o derecho ${ }^{6}$. Los túneles que se abren hacia el ventrículo derecho se visualizan en el eje corto paraesternal de la válvula aórtica. La función del ventrículo izquierdo, que se afecta de forma variable con hipertrofia y dilatación, se evalúa en la vista paraesternal en el eje corto a nivel de los músculos papilares ${ }^{6}$. En casos no concluyentes, en los que no se puedan demostrar con claridad las arterias coronarias, se puede requerir angiografía con catéter o resonancia magnética.

\section{Caso clínico}

Lactante de sexo femenino, de 3 meses, con polipnea desde el nacimiento y empeoramiento del cuadro de curso progresivo. En la exploración física se presenta irritable, con palidez moderada, frecuencia cardiaca de 160 latidos por minuto, frecuencia respiratoria de 80 por minuto, tiraje subcostal e intercostal, primer ruido cardiaco normal, segundo ruido cardiaco aumentado, soplo sistodiastólico II-III/VI en el segundo espacio intercostal izquierdo, latido epigástrico, precordio hiperdinámico, pulsos presentes simétricos amplios, e hígado a $4 \mathrm{~cm}$ debajo del reborde costal derecho.

En la radiografía de tórax se evidenció cardiomegalia y crecimiento de cavidades derechas con signos de hiperflujo pulmonar. El electrocardiograma mostró hipertrofia ventricular derecha y sobrecarga de volumen. El ecocardiograma transtorácico en el eje largo paraesternal mostró un flujo que se originaba a nivel de la coronaria izquierda y se presentaba durante la diástole con dirección hacia el ventrículo derecho. Se evidenció, además, arteria pulmonar y ramas dilatadas. En el eje apical de cinco cámaras se observa el trayecto del túnel de la aorta al ventrículo derecho (Fig. 1). La anatomía coronaria fue normal. Presentaba, además, un ductus arterioso persistente pequeño.

Se inició tratamiento para insuficiencia cardiaca y se decidió realizar cateterismo cardiaco diagnóstico y terapéutico, el cual mostró un patrón coronario normal. En un aortograma en incidencia oblicua anterior derecha se observó una estructura vascular externa originada en la raíz de la aorta, que correspondía al túnel aorto-ventricular (Fig. 2).

Se realizaron dos procedimientos, el primero una angiografía diagnóstica y el segundo una intervención percutánea para la colocación transcatéter de un dispositivo para cerrar el túnel. El aortograma ascendente, en vista oblicua anterior izquierda, mostró un túnel con una dilatación tortuosa en el lado derecho del arco aórtico, que drenaba en la región trabecular del ventrículo derecho. El diámetro medido fue de $6 \mathrm{~mm}$, con una medida aórtica de $7 \mathrm{~mm}$. Principio del formulario. 


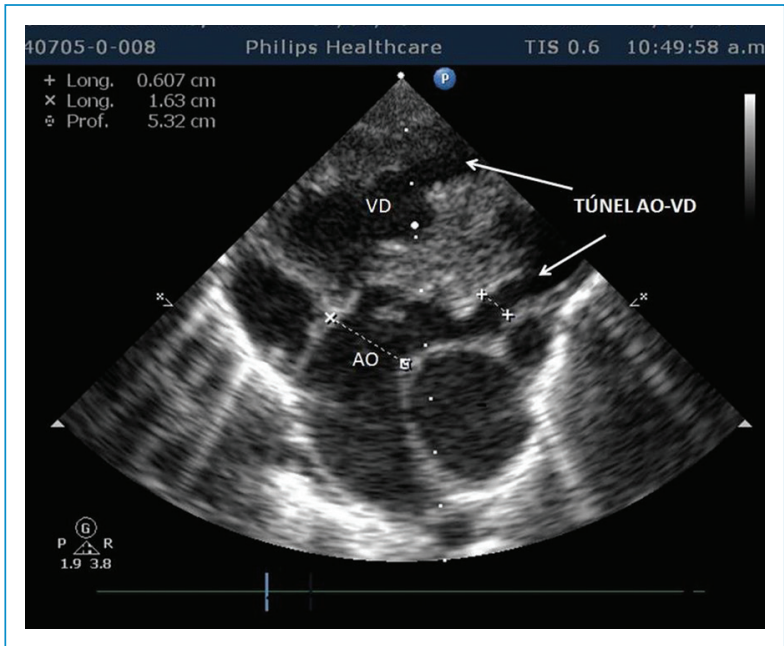

Figura 1. Ecocardiograma transtorácico: eje apical de cinco cámaras. Se observa el túnel de la aorta (AO) al ventrículo derecho (VD).

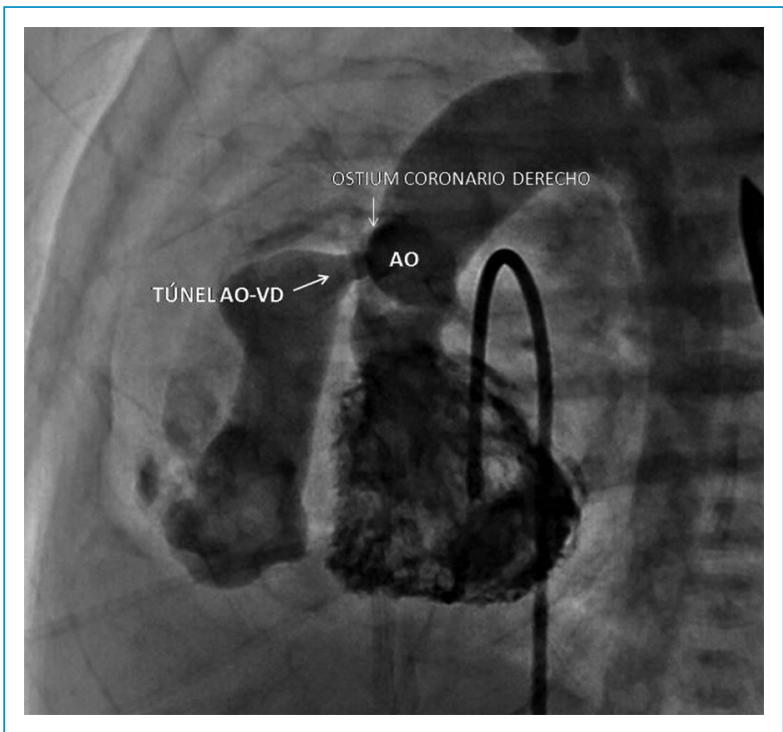

Figura 2. Imágenes fijas de angiografía. En proyección lateral se observa el origen del túnel en estrecha proximidad con el ostium coronario derecho. A0: aorta; VD: ventrículo derecho.

Se procedió a cateterismo inguinal derecho de forma habitual para el acceso venoso y cateterismo inguinal izquierdo para el acceso arterial. Posteriormente, se realizó angiografía biplanar para lograr la opacificación del túnel, determinándose el diámetro en $6 \mathrm{~mm}$, con boca aórtica de $7 \mathrm{~mm}$. Se preparó el dispositivo de liberación y se usó guía de intercambio de 0.035

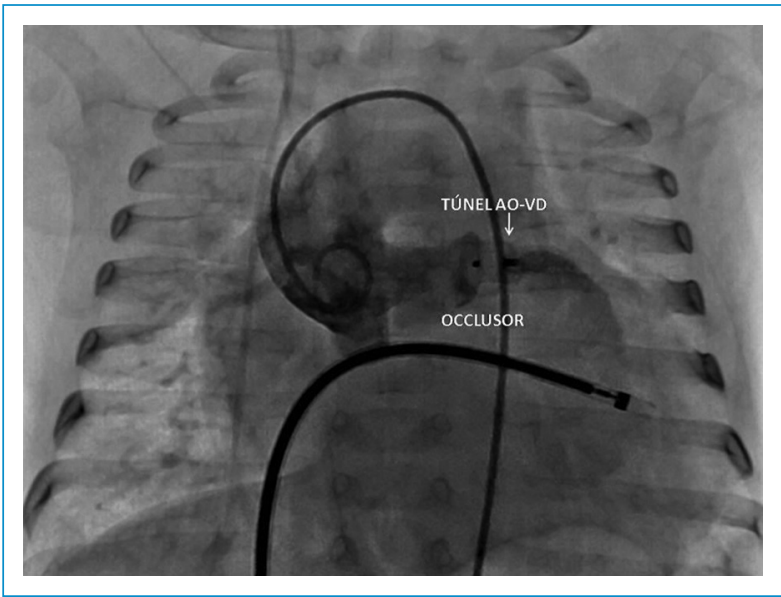

Figura 3. Aortografía en proyección frontal en la que se evidencia el oclusor ubicado en posición correcta. AO: aorta; VD: ventrículo derecho.

pulgadas $(0.89 \mathrm{~mm})$ con punta en $\mathrm{J}$, introduciéndose el dilatador y la vaina de liberación sobre la guía con punta en $\mathrm{J}$ en la aorta. Así, se confirmó la ubicación con inyección manual de medio de contraste. Una vez verificada la correcta posición, se retiraron el dilatador y la guía con punta en J. Con el dispositivo oclusor ya preparado y acoplada la válvula hemostática, se procedió a introducir el cable de liberación a través de la misma junto al cargador, el cual se conecta a la vaina de liberación; se procedió a liberar el faldón de retención en la ampolla del túnel. Mediante angiografía se verificó la posición y se procedió a desplegar el oclusor en el túnel; se verificó de nuevo la posición del oclusor, se determinó la presencia de cortocircuito residual y se culminó con la liberación del dispositivo. Una vez liberado el oclusor, se realizó un nuevo angiograma para comprobar la posición correcta (Fig. 3).

Para el procedimiento se usó un dispositivo de malla de nitinol autoexpandible. La configuración del dispositivo es una cintura central con dos discos de retención. La cintura central está diseñada para llenar el defecto. No hubo complicaciones durante el procedimiento, por cuanto se consideró como exitoso. El ductus permeable fue tratado en una segunda oportunidad por intervencionismo. En el ecocardiograma de seguimiento a los 24 meses se encontró un grado moderado de insuficiencia aórtica sin sintomatología evidente.

\section{Discusión}

El túnel aorto-ventricular es una cardiopatía congénita poco frecuente. Se trata de un canal extracardiaco 


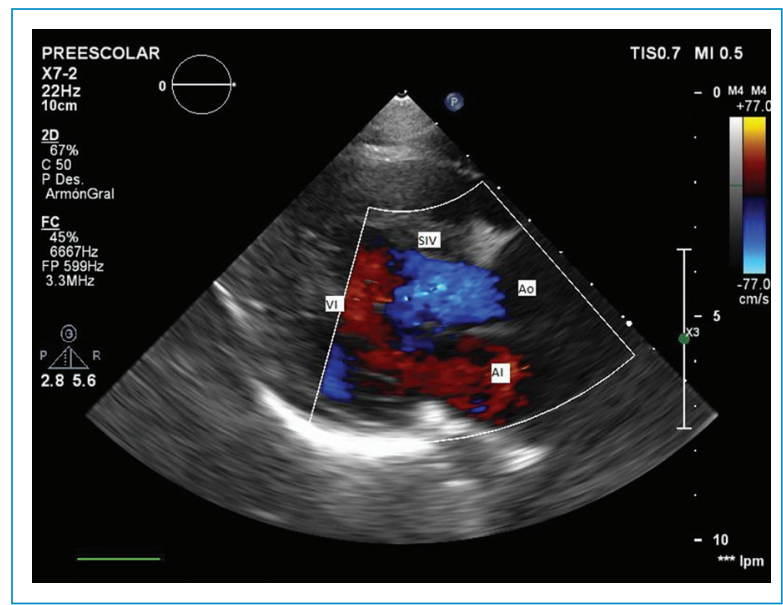

Figura 4. Ecocardiograma posprocedimiento. No se observa flujo residual, lo que confirma la oclusión completa del TAVD. Al: aurícula izquierda; Ao: aorta; TAVD: túnel aorto ventricular derecho; SIV: septum interventricular; VI: ventrículo izquierdo.

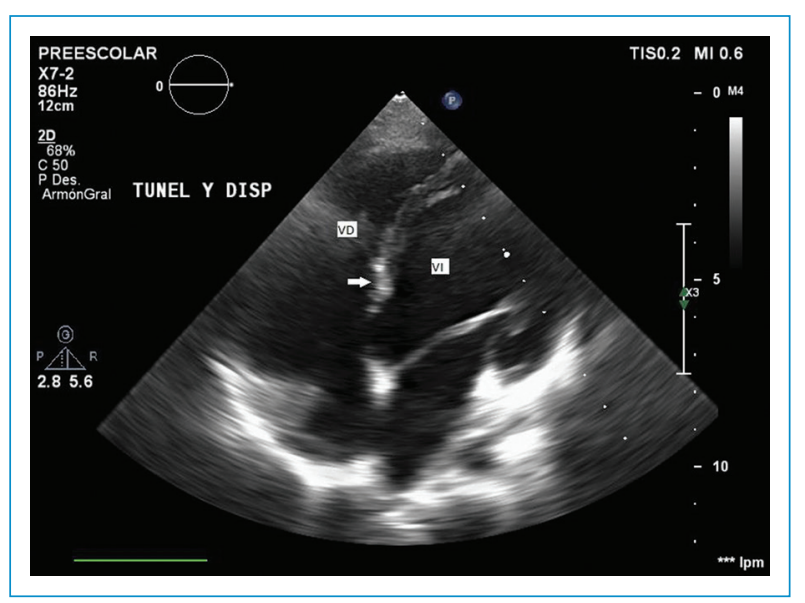

Figura 5. Ecocardiografía de control. El dispositivo se mantiene en posición adecuada. Flecha en blanco señala al oclusor localizado en el TAVD sin pase de flujo 3. TAVD: túnel aorto ventricular derecho. VD: ventrículo derecho; VI: ventrículo izquierdo.

que conecta la aorta ascendente encima de la unión sinotubular a la cavidad ventricular izquierda 0 , menos común, al ventrículo derecho. Aproximadamente se han reportado 130 casos en la literatura. La condición fue descrita por primera vez por Levy en 1963, en tres pacientes con estudio patológico ${ }^{5}$. El estudio con mayor información fue realizado por Martins, et al. ${ }^{7}$, quienes identificaron 11 pacientes entre 1963 y agosto de 2002, con predominio del sexo masculino. En dicha serie, ocho pacientes menores de 6 meses desarrollaron insuficiencia cardiaca congestiva y el resto estuvieron clínicamente asintomáticos. Todos los pacientes presentaban datos de regurgitación aórtica leve.

El túnel aorto-ventricular derecho es una vía anormal que tiene su orificio por encima o en la porción superior derecha del seno de Valsalva, justo a la izquierda del orificio de la arteria coronaria derecha, y que entra en el infundíbulo del ventrículo derecho. Las cardiopatías asociadas a esta anomalía incluyen defecto septal interventricular, estenosis aórtica y subaórtica crítica, y fístula coronaria derecha al túnel aorto-ventricular. Desde el punto de vista histopatológico, el extremo arterial del túnel es similar a la aorta, con tejido fibroso, fibras elásticas y células de músculo liso, mientras que el extremo ventricular contiene colágeno hialinizado y músculo. Dentro del propio túnel puede haber una unión bien definida entre los componentes ventriculares y arteriales. En cuanto a la hemodinamia, la anomalía produce un cortocircuito de izquierda a derecha con sobrecarga de presión y de volumen en el ventrículo derecho ${ }^{8}$.

Algunos pacientes presentan un soplo cardiaco asintomático, pero es frecuente la insuficiencia cardiaca en el primer año de vida. La gravedad y la progresión de la insuficiencia cardiaca son, sin embargo, bastante variables y van desde muchos años de compensación asintomática ${ }^{9-11}$ hasta una descompensación rápida, muerte súbita o incluso muerte intrauterina. En la paciente del caso, el curso de la insuficiencia cardiaca fue progresivo y hubo descompensación rápida.

El estudio diagnóstico de elección es el ecocardiograma transtorácico, en modo Doppler color, que muestra un flujo diastólico dirigiéndose hacia el ventrículo derecho junto con alteraciones en la arteria pulmonar. La ecocardiografía puede establecer un diagnóstico certero mediante la demostración de los dos extremos del túnel que conecta la aorta. De todas estas características, la dilatación de la aorta ascendente extensa y uniforme puede ser el mejor signo no invasivo para el diagnóstico de túnel aorto-ventricular derecho, pero no siempre está presente en forma temprana ${ }^{8}$.

El ecocardiograma inicial de la paciente mostró una gran dilatación de la arteria pulmonar con un flujo diastólico originado a nivel de la valva coronariana derecha hacia el ventrículo derecho. Ante la duda diagnóstica, fue llevada a cateterismo cardiaco. El diagnóstico diferencial incluye defecto septal ventricular con lesión valvular, doble lesión aórtica, aneurisma y rotura del seno de Valsalva ${ }^{12}$. 
Para el tratamiento de esta cardiopatía se recomienda el cierre quirúrgico en el momento del diagnóstico, independientemente de la edad y de los síntomas, debido a la poca efectividad del tratamiento médico para estabilizar los síntomas y al riesgo de desarrollar insuficiencia aórtica grave si se atiende de manera tardía? ${ }^{7}$ La reparación del túnel aorto-ventricular derecho debe llevarse a cabo lo antes posible para minimizar los daños al ventrículo y evitar el desarrollo de enfermedad vascular pulmonar obstructiva ${ }^{12}$.

Aunque el cierre quirúrgico del túnel es la base del tratamiento para esta anomalía, tiene una alta mortalidad quirúrgica. El objetivo de cualquier tratamiento es obliterar el túnel. Las técnicas que se han descrito son el cierre del orificio de la aorta con o sin un parche, el cierre del extremo ventricular del túnel, la obliteración del túnel (ligadura del túnel o resección parcial del túnel) y el cierre con doble parche de ambos orificios (aórtico y ventricular) ${ }^{13}$.

Otra opción de tratamiento que puede ser aplicable a estos pacientes es el cierre con un dispositivo de cierre percutáneo en pacientes seleccionados sin otras afecciones asociadas $^{12}$. El cierre con dispositivo por intervencionismo ha sido reportado por Poptani, et al. ${ }^{14}$ y Chessa, et al. ${ }^{15}$. El caso presentado es el tercero realizado en el mundo y el primero en Latinoamérica, y ha tenido resultado exitoso.

Las lesiones asociadas pueden tratarse por separado o en el momento de la reparación del túnel aorto-ventricular. La paciente presentaba un ductus permeable que fue tratado en una segunda oportunidad por intervencionismo. En el ecocardiograma de seguimiento a los 24 meses se encontró un grado moderado de insuficiencia aórtica, sin sintomatología evidente.

En conclusión, el cierre del túnel aorto-ventricular derecho utilizando un dispositivo de cierre percutáneo desde el final de la aorta es factible y puede ser considerado una alternativa al cierre quirúrgico abierto.

\section{Responsabilidades éticas}

Protección de personas y animales. Los autores declaran que para esta investigación no se han realizado experimentos en seres humanos ni en animales.
Confidencialidad de los datos. Los autores declaran que han seguido los protocolos de su centro de trabajo sobre la publicación de datos de pacientes.

Derecho a la privacidad y consentimiento informado. Los autores han obtenido el consentimiento informado de los pacientes y/o sujetos referidos en el artículo. Este documento obra en poder del autor de correspondencia.

\section{Conflicto de intereses}

Los autores declaran que no existen conflictos de intereses.

\section{Agradecimientos}

Los autores agradecen a los doctores Gustavo Miranda y Haylín Loo su participación en el manejo del caso.

\section{Bibliografía}

1. Bharati S, Lev M, Cassels D. Aortic-right ventricular tunnel. Chest J 1973;63:198-202.

2. Saylam A, Tuncali T, Ikizler C, Aytac A. Aorto-right ventricular tunnel: a new concept in congenital cardiac malformations. Ann Thorac Surg. 1974;18:634-7.

3. Somerville J, English T, Ross DN. Aorto-left ventricular tunnel. Clinical features and surgical management. Br Heart J. 1974;36:321-8.

4. Amoozgar H, Shakibazad N, Amirghofran AA. Aneurysmal aorto-left ventricular tunnel. Int Cardiovasc Res J. 2012;6:30-2.

5. Levy MJ, Lillehei CW, Anderson RC, Amplatz K, Edwards JE. Aortic-left ventricular tunnel. Circulation. 1963;27:841-53.

6. Mueller C, Dave H, Pretre R. Surgical repair of aortoventricular tunnel. Multimed Man of Cardiothorac Surg. 2012;2012:mms006.

7. Martins JD, Sherwood MC, Mayer JE, Keane JF. Aortic-left ventricular tunnel: 35-year experience. J Am Coll Cardiol. 2004;44:446-50.

8. Van Son JA, Hambsch J, Schneider P, Mohr FW. Repair of aortic-right ventricular tunnel. Eur J Cardiothorac Surg. 1998:14:214-7.

9. Colak N, Nazli Y, Alpay MF, Cakir O. Aorto-left ventricular tunnel with its origin in the left sinus of Valsalva associated with a single coronary artery and aortic insufficiency in an adult. J Card Surg. 2011;26:437-9.

10. Burkhardt BE, Arnold R, Schlensak C, Bökenkamp R, Stiller B. Aorto-right ventricular tunnel causing functional tricuspid atresia. Circulation. 2012;125:e591-4.

11. Talwar S1, Nair VV, Kothari SS, Gulati GS, Choudhary SK, Airan B. Aortic-right ventricular tunnel with anomalous right coronary artery. J Card Surg. 2011;26:521-6.

12. Chessa M, Chaudhari M, De Giovanni JV. Aorto-left ventricular tunnel: transcatheter closure using an Amplatzer duct occluder device. Am J Cardiol. 2000;86:253.

13. Mitropoulos FA, Laks $\mathrm{H}$, Kanakis MA, Levi D. Aorto-left ventricular tunnel: an alternative surgical approach. Ann Thoracic Surg. 2006;82:1113-5.

14. Poptani VA, Thakkar BM, Patel NH. Transcatheter closure of a rare case of aorto-right ventricular tunnel with single coronary artery. J Invasive Cardiol. 2010;22:611-4.

15. Chessa M, Chaudhari M, De Giovanni JV. Aorto-left ventricular tunnel: transcatheter closure using Amplatzer duct occlude device. Am J Cardiol. 2000;86:253-4. 